\title{
Extrapolation for meson screening masses from imaginary to real chemical potential
}

\author{
Masahiro Ishii, ${ }^{1, *}$ Akihisa Miyahara, ${ }^{1, \dagger}$ Hiroaki Kouno, ${ }^{2, *}$ and Masanobu Yahiro ${ }^{1, \S}$ \\ ${ }^{1}$ Department of Physics, Graduate School of Sciences, Kyushu University, Fukuoka 819-0395, Japan \\ ${ }^{2}$ Department of Physics, Saga University, Saga 840-8502, Japan
}

(Received 26 July 2018; published 18 June 2019)

\begin{abstract}
We first extend our formulation for the calculation of $\pi$ - and $\sigma$-meson screening masses to the case of finite chemical potential $\mu$. We then consider the imaginary- $\mu$ approach, which is an extrapolation method from an imaginary chemical potential $\left(\mu=i \mu_{\mathrm{I}}\right)$ to a real one $\left(\mu=\mu_{\mathrm{R}}\right)$. The feasibility of the method is discussed based on the entanglement Polyakov-loop extended Nambu-Jona-Lasinio (EPNJL) model in 2-flavor system. As an example, we investigate how reliable the imaginary- $\mu$ approach is for $\pi$ - and $\sigma$-meson screening masses, comparing "screening masses at $\mu_{\mathrm{R}}$ in the method" with "those calculated directly at $\mu_{\mathrm{R}}$." We finally propose a new extrapolation method and confirm its efficiency.
\end{abstract}

DOI: $10.1103 /$ PhysRevD.99.114010

\section{INTRODUCTION}

$T$ and $\mu$ dependence of hadron masses are closely related with those of the ground-state structure of hot QCD matter, where $T$ is temperature and $\mu$ means quark-number chemical potential. In fact, medium modification of vector and $\eta^{\prime}$ mesons has been measured in heavy-ion collisions $[1,2]$. These results indicate the chiral and the effective $U(1)_{\mathrm{A}}$-symmetry restoration. It is, therefore, important to determine $T$ and $\mu$ dependence of light hadron masses.

Lattice QCD (LQCD) is a powerful tool to investigate the QCD matter at finite $T$ and $\mu$. In fact, many LQCD calculations have been done for low density $(\mu / T \lesssim 1)$. The calculation in the high density region is still challenging because of the well-known "sign problem." Several methods were proposed so far to circumvent the sign problem; the Taylor expansion method [3,4], the reweighting method [5], the imaginary- $\mu$ method [6-9], the canonical approach [10], the complex Langevin method [11-14], and the Lefschetz thimble theory $[15,16]$. These have made great progress, but all the results are consistent only in $\mu / T \lesssim 1$ at the present stage. Among them, we pick up the imaginary- $\mu$ method in the present paper. When one considers $\mu$ as a complex variable, this method corresponds to the analytic

\footnotetext{
*ishii@phys.kyushu-u.ac.jp

miyahara@email.phys.kyushu-u.ac.jp

*kounoh@cc.saga-u.ac.jp

§yahiro@phys.kyushu-u.ac.jp
}

Published by the American Physical Society under the terms of the Creative Commons Attribution 4.0 International license. Further distribution of this work must maintain attribution to the author(s) and the published article's title, journal citation, and DOI. Funded by SCOAP ${ }^{3}$. continuation from the imaginary chemical potential $\left(\mu=i \mu_{\mathrm{I}}\right)$ to the real one $\left(\mu=\mu_{\mathrm{R}}\right)$.

In LQCD simulation for finite $\theta \equiv \mu_{\mathrm{I}} / T$, the thermodynamic potential $\Omega_{\mathrm{QCD}}(\theta)$ has the Roberge and Weiss (RW) periodicity: $\Omega_{\mathrm{QCD}}(\theta)=\Omega_{\mathrm{QCD}}(\theta+2 \pi / 3)$ [17]. The $\mathrm{QCD}$ phase diagram has the first-order phase transition (RW phase transition) at $T \geq T_{\mathrm{RW}}$ and $\theta=\pi / 3$, where $T_{\mathrm{RW}}$ is $\mathrm{RW}$ transition temperature. The endpoint of RW phase transition is located at $(\theta, T)=\left(\pi / 3, T_{\mathrm{RW}}\right)$. The order of the RW endpoint and the value of $T_{\mathrm{RW}}$ have been investigated in 2-flavor LQCD simulations [6-8].

One can consider effective models as a complementary approach to the first-principle LQCD simulation. The Polyakov-loop extended Nambu-Jona-Lasinio (PNJL) model [18-38] qualitatively reproduces 2-flavor LQCD data in $\mu_{\mathrm{R}} / T \lesssim 1$, since the model can treat the chiral and the deconfinement transition simultaneously. In addition, the model is successful in accounting for 2-flavor LQCD data in $0 \leq \theta \lesssim \pi / 3[34,35]$, because it has the RW periodicity. The entanglement PNJL (EPNJL) model [39-41] is an improved version of the PNJL model. The EPNJL model quantitatively reproduces 2-flavor LQCD data in $0 \leq \theta \lesssim \pi / 3$ [39] and $\mu_{\mathrm{R}} / T \lesssim 1$ [40], since the model possesses the RW periodicity and the strong correlation between the chiral and the deconfinement transition.

Meson masses can be classified into "meson pole mass" and "meson screening mass." In LQCD simulations at finite $T$, the derivation of meson screening mass is easier than that of meson pole mass, since the spatial lattice size is larger than the temporal one; see Appendix of Ref. [42] for the further explanation. Meanwhile, in NJL-type effective models, time-consuming calculations were needed for the meson screening mass compared with that of the meson 
pole mass. Recently, this problem was solved by our previous works [42-44] for the case of $\mu=0$.

In this paper, for simplicity, we concentrate on the $\pi$-meson and $\sigma$-meson screening masses, $M_{\pi}^{\text {scr }}$ and $M_{\sigma}^{\text {scr }}$, in the framework of 2-flavor EPNJL model. We apply the method of Refs. [42-44] for the case of finite $\mu_{\mathrm{R}}$ and $\mu_{\mathrm{I}}$, and then investigate how reliable the imaginary- $\mu$ method is for $M_{\pi}^{\text {scr }}$ and $M_{\sigma}^{\text {scr }}$. For this purpose, we compare "the $M_{\xi}^{\text {scr }}$ extrapolated from $i \mu_{\mathrm{I}}$ (extrapolating result)" with "the $M_{\xi}^{\text {scr }}$ calculated directly at $\mu_{\mathrm{R}}$ (direct result)" for $\xi=\pi, \sigma$ mesons.

In Sec. II, we explain a way of calculating the meson screening mass at finite $\mu$. Numerical results are shown in Sec. III. Section IV is devoted to a summary.

\section{FORMALISM}

\section{A. Model setting}

The Lagrangian density of 2-flavor EPNJL model is defined by

$$
\begin{aligned}
\mathcal{L}= & \bar{\psi}\left(i \gamma_{\nu} D^{\nu}-m_{0}\right) \psi+G_{\mathrm{S}}(\Phi)\left[(\bar{\psi} \psi)^{2}+\left(\bar{\psi} i \gamma_{5} \vec{\tau} \psi\right)^{2}\right] \\
& -\mathcal{U}(\Phi[A], \bar{\Phi}[A], T)
\end{aligned}
$$

with u- and d-quark fields $\psi=(u, d)^{\mathrm{T}}$ and the isospin matrix $\vec{\tau}$. We assume isospin symmetry, i.e., u and d quarks have the same mass $m_{0}$. The gluon field $A^{\nu}$ is introduced through the covariant derivative $D^{\nu}=\partial^{\nu}+i A^{\nu}$ with $A^{\nu}=\delta_{0}^{\nu} g\left(A^{0}\right)_{a} \lambda_{a} / 2=-\delta_{0}^{\nu} i g\left(A_{4}\right)_{a} \lambda_{a} / 2$, where the matrices $\lambda_{a}$ are the Gell-Mann matrices in color space and $g$ is the gauge coupling. Here, we consider only the time component $A_{4}$ of $A_{\nu}$ and assume that the $A_{4}$ is a homogeneous and static background field.

In the EPNJL model, the Polyakov loop $\Phi$ and its Hermitian conjugate $\bar{\Phi}$ are defined by

$$
\Phi=\frac{1}{3} \operatorname{tr}_{\mathrm{c}}(L), \quad \bar{\Phi}=\frac{1}{3} \operatorname{tr}_{\mathrm{c}}\left(L^{*}\right)
$$

with $L=\exp \left[i A_{4} / T\right]=\exp \left[i \operatorname{diag}\left(A_{4}^{11}, A_{4}^{22}, A_{4}^{33}\right) / T\right]$ for real classical variables $A_{4}^{j j}(j=1,2,3)$. The trace $\operatorname{tr}_{\mathrm{c}}$ is taken in color space. The relation between $A_{4}^{j j}$ and $\Phi$ or $\bar{\Phi}$ is summarized in the Appendix. The coupling constant $G_{\mathrm{S}}$ of the four-quark interaction is assumed to depend on the Polyakov loop $\Phi$ and $\bar{\Phi}$

$$
G_{\mathrm{S}}(\Phi)=G_{\mathrm{S}}(0) \times\left[1-\alpha_{1} \Phi \bar{\Phi}-\alpha_{2}\left(\Phi^{3}+\bar{\Phi}^{3}\right)\right],
$$

which is essential to reproduce the strong correlation between the chiral and deconfinement transitions observed in LQCD simulations [45-47].

The Polyakov loop $\Phi$ and its Hermitian conjugate $\bar{\Phi}$ are mainly governed by the Polyakov-loop potential $\mathcal{U}$ in Eq. (1). We use the logarithm-type Polyakov-loop potential
$\mathcal{U}$ of Ref. [26]. The parameter set in $\mathcal{U}$ is determined from LQCD data on thermodynamic quantities in the pure gauge limit. The $\mathcal{U}$ has one dimensionful parameter $T_{0}$ and the value is often set to $T_{0}=270 \mathrm{MeV}$ since the deconfinement transition occurs at $T=270 \mathrm{MeV}$ in the pure gauge limit. When one considers the dynamical quarks, the typical energy scale $T_{0}$ depends on the number of flavors $\left(N_{\mathrm{f}}\right)$. Hence we treat $T_{0}$ as an adjustable parameter and determine the value to reproduce the chiral- and deconfinement-transition temperatures $T_{\mathrm{c}}^{\chi, \mathrm{LQCD}}=173 \pm 8 \mathrm{MeV}$ and $T_{\mathrm{c}}^{\mathrm{d}, \mathrm{LQCD}}=173 \pm 8 \mathrm{MeV}$ calculated with 2-flavor LQCD simulations at zero chemical potential [45-47].

Applying the mean field approximation to Eq. (1) leads to the linearized Lagrangian density

$$
\mathcal{L}^{\mathrm{MFA}}=\bar{\psi} S^{-1} \psi-G_{\mathrm{S}}(\Phi) \sigma^{2}-\mathcal{U}(\Phi[A], \bar{\Phi}[A], T),
$$

where the dressed quark propagator $S$ is defined by

$$
S=\frac{1}{i \gamma_{\nu} \partial^{\nu}-i \gamma_{0} A_{4}-M}
$$

with the effective quark mass $M=m_{0}-2 G_{\mathrm{S}}(\Phi) \sigma$ and the chiral condensate $\sigma=\langle\bar{\psi} \psi\rangle$. One can make the path integral over the quark fields analytically, and the thermodynamic potential $\Omega$ per unit volume is obtained by

$$
\begin{aligned}
\Omega= & U_{\mathrm{M}}+\mathcal{U}-2 N_{\mathrm{f}} \int \frac{d^{3} \boldsymbol{p}}{(2 \pi)^{3}} \\
& \times\left[3 E_{p}+\frac{1}{\beta} \ln \left[1+3\left(\Phi+\bar{\Phi} e^{-\beta\left(E_{p}-\mu\right)}\right) e^{-\beta\left(E_{p}-\mu\right)}+e^{-3 \beta\left(E_{p}-\mu\right)}\right]\right. \\
& \left.+\frac{1}{\beta} \ln \left[1+3\left(\bar{\Phi}+\Phi e^{-\beta\left(E_{p}+\mu\right)}\right) e^{-\beta\left(E_{p}+\mu\right)}+e^{-3 \beta\left(E_{p}+\mu\right)}\right]\right]
\end{aligned}
$$

with $E_{\boldsymbol{p}}=\sqrt{\boldsymbol{p}^{2}+M^{2}}$ and $U_{\mathrm{M}}=G_{\mathrm{S}}(\Phi) \sigma^{2}$. The meanfield variables $\sigma, \Phi, \bar{\Phi}$ are determined so as to minimize the potential $\Omega$. For real $\mu$, we take the approximation $\Phi=\bar{\Phi}$ for simplicity. This approximation is pretty good for $\mu_{\mathrm{R}} / T \lesssim 1$ and not so bad even for $\mu_{\mathrm{R}} / T \gtrsim 1$ [38].

In the $\mu_{\mathrm{I}}$ region, this thermodynamic potential $\Omega$ has the RW periodicity $[32,33,37]$. The RW periodicity stems from the fact that $\Omega$ is invariant under the extended $Z_{3}$ transformation [37] defined by

$$
\Phi \rightarrow e^{-i 2 \pi k / 3} \Phi, \quad \bar{\Phi} \rightarrow e^{i 2 \pi k / 3} \bar{\Phi}, \quad \theta \rightarrow \theta+\frac{2 \pi k}{3}
$$

for integer $k$.

The three-dimensional momentum $\boldsymbol{p}$ integral in Eq. (6) has ultraviolet divergence and needs to be regularized. In this paper, we use the Pauli-Villars $(\mathrm{PV})$ regularization $[48,49]$. When $\Omega$ is divided into $\Omega=U_{\mathrm{M}}+\mathcal{U}+\Omega_{\mathrm{F}}(M)$, the function $\Omega_{\mathrm{F}}(M)$ is regularized in the PV scheme as 
TABLE I. Model parameters determined from physical observables at vacuum.

\begin{tabular}{lcc}
\hline \hline$m_{0}[\mathrm{MeV}]$ & $\Lambda[\mathrm{MeV}]$ & $G_{\mathrm{S}}(0) \Lambda^{2}$ \\
\hline 6.3 & 768 & 2.95 \\
\hline
\end{tabular}

$$
\Omega_{\mathrm{F}}^{\mathrm{reg}}(M)=\sum_{\alpha=0}^{2} C_{\alpha} \Omega_{\mathrm{F}}\left(M_{\alpha}\right),
$$

where $M_{0}=M$ and the $M_{\alpha}(\alpha=1,2)$ mean masses of auxiliary particles. The parameters $M_{\alpha}$ and $C_{\alpha}$ are determined so as to satisfy the condition $\sum_{\alpha=0}^{2} C_{\alpha}=$ $\sum_{\alpha=0}^{2} C_{\alpha} M_{\alpha}^{2}=0$ in order to remove the quartic, the quadratic, and the logarithmic divergence in $\Omega_{\mathrm{F}}$. We then set $\left(C_{0}, C_{1}, C_{2}\right)=(1,-2,1)$ and $\left(M_{0}^{2}, M_{1}^{2}, M_{2}^{2}\right)=\left(M^{2}, M^{2}+\Lambda^{2}\right.$, $\left.M^{2}+2 \Lambda^{2}\right)$. The parameter $\Lambda$ should be finite even after the regularization [49], since the present model is nonrenormalizable.

The EPNJL model has three parameters $m_{0}, G_{\mathrm{S}}(0), \Lambda$ in addition to $T_{0}, \alpha_{1}, \alpha_{2}$. We set $m_{0}$ to $m_{0}=6.3 \mathrm{MeV}$ and determine $G_{\mathrm{S}}(0), \Lambda$ to reproduce the experimental values of pion mass $M_{\pi}=138 \mathrm{MeV}$ and its decay constant $f_{\pi}=93.3 \mathrm{MeV}$ at vacuum. The values of $m_{0}, G_{\mathrm{S}}(0), \Lambda$ are summarized in Table I. The remaining parameters $T_{0}, \alpha_{1}, \alpha_{2}$ are determined from LQCD results on the chiral- and deconfinement-transition temperatures [45-47]; see Sec. III A for the determination of the parameters $T_{0}, \alpha_{1}, \alpha_{2}$.

\section{B. Meson screening mass at finite $\boldsymbol{T}$ and $\boldsymbol{\mu}$}

Following the previous work [43], we first consider $\pi$ and $\sigma$ mesons at $T=\mu=0$. The current operator is expressed by

$$
J_{\xi}(x)=\bar{\psi}(x) \Gamma_{\xi} \psi(x)-\left\langle\bar{\psi}(x) \Gamma_{\xi} \psi(x)\right\rangle
$$

with $x=(t, \boldsymbol{x})$ for meson species $\xi=\pi, \sigma$, where $\Gamma_{\sigma}=1$ for $\sigma$ meson and $\Gamma_{\pi}=i \gamma_{5} \tau_{3}$ for $\pi$ meson. The mesonic correlation function in coordinate space is defined by

$$
\zeta_{\xi \xi}(t, \boldsymbol{x}) \equiv\left\langle 0\left|\mathrm{~T}\left(J_{\xi}(t, \boldsymbol{x}) J_{\xi}^{\dagger}(0)\right)\right| 0\right\rangle .
$$

Here, the symbol $\mathrm{T}$ stands for the time-ordered product. The Fourier transform $\chi_{\xi \xi}\left(q_{0}^{2}, \boldsymbol{q}^{2}\right)$ of $\zeta_{\xi \xi}(t, \boldsymbol{x})$ is obtained by

$$
\chi_{\xi \xi}\left(q_{0}^{2}, \tilde{q}^{2}\right)=i \int d^{4} x e^{i q \cdot x} \zeta_{\xi \xi}(t, \boldsymbol{x})
$$

for an external momentum $q=\left(q_{0}, \boldsymbol{q}\right)$ and $\tilde{q}= \pm|\boldsymbol{q}|$. When we take the random-phase approximation, we can get $\chi_{\xi \xi}$ as

$$
\chi_{\xi \xi}=\frac{\Pi_{\xi}}{1-2 G_{\mathrm{S}}(\Phi) \Pi_{\xi}}
$$

for $\xi=\pi, \sigma$. The one-loop polarization function $\Pi_{\xi}$ is explicitly calculated by

$$
\begin{aligned}
\Pi_{\sigma} & =(-2 i) \int \frac{d^{4} p}{(2 \pi)^{4}} \operatorname{tr}_{\mathrm{c}, \mathrm{d}}(i S(p+q) i S(p)) \\
& =4 i\left[I_{1}+I_{2}-\left(q^{2}-4 M^{2}\right) I_{3}\right]
\end{aligned}
$$

for $\sigma$ meson and

$$
\begin{aligned}
\Pi_{\pi} & =(-2 i) \int \frac{d^{4} p}{(2 \pi)^{4}} \operatorname{tr}_{\mathrm{c}, \mathrm{d}}\left(\left(i \gamma_{5}\right) i S(p+q)\left(i \gamma_{5}\right) i S(p)\right) \\
& =4 i\left[I_{1}+I_{2}-q^{2} I_{3}\right]
\end{aligned}
$$

for $\pi$ meson, where the trace $\operatorname{tr}_{\mathrm{c}, \mathrm{d}}$ is taken in color and Dirac spaces. Three functions in Eqs. (13) and (14) are defined by

$$
\begin{gathered}
I_{1}=\int \frac{d^{4} p}{(2 \pi)^{4}} \operatorname{tr}_{\mathrm{c}}\left[\frac{1}{p^{2}-M^{2}}\right], \\
I_{2}=\int \frac{d^{4} p}{(2 \pi)^{4}} \operatorname{tr}_{\mathrm{c}}\left[\frac{1}{(p+q)^{2}-M^{2}}\right], \\
I_{3}=\int \frac{d^{4} p}{(2 \pi)^{4}} \operatorname{tr}_{\mathrm{c}}\left[\frac{1}{\left(p^{2}-M^{2}\right)\left((p+q)^{2}-M^{2}\right)}\right] .
\end{gathered}
$$

These functions are regularized with the same procedure as shown in Eq. (8).

In the two cases of (a) finite $T$ and $\mu=\mu_{\mathrm{R}}$ and (b) finite $T$ and $\mu=i \mu_{\mathrm{I}}$, one can get the final equations by taking the following replacement

$$
\begin{aligned}
p_{0} & \rightarrow i \omega_{n}+i A_{4}+\mu=i(2 n+1) \pi T+i A_{4}+\mu, \\
\int \frac{d^{4} p}{(2 \pi)^{4}} & \rightarrow i T \sum_{n=-\infty}^{\infty} \int \frac{d^{3} \boldsymbol{p}}{(2 \pi)^{3}} .
\end{aligned}
$$

The meson screening mass $M_{\xi}^{\text {scr }}$ for $\xi$ meson is defined by

$$
M_{\xi}^{\mathrm{scr}}=-\lim _{r=|x| \rightarrow \infty}\left(\frac{d}{d r} \ln \zeta_{\xi \xi}(0, \boldsymbol{x})\right),
$$

where the correlation function $\zeta_{\xi \xi}(0, x)$ in coordinate space is obtained by the Fourier transformation of the correlation function $\chi_{\xi \xi}\left(0, \tilde{q}^{2}\right)$ in momentum space as

$$
\zeta_{\xi \xi}(0, \boldsymbol{x})=\frac{1}{4 \pi^{2} i r} \int_{-\infty}^{\infty} d \tilde{q} \tilde{q} \chi_{\xi \xi}\left(0, \tilde{q}^{2}\right) e^{i \tilde{q} r}
$$

see Fig. 1 to understand the meaning of $\tilde{q}$ integral. 


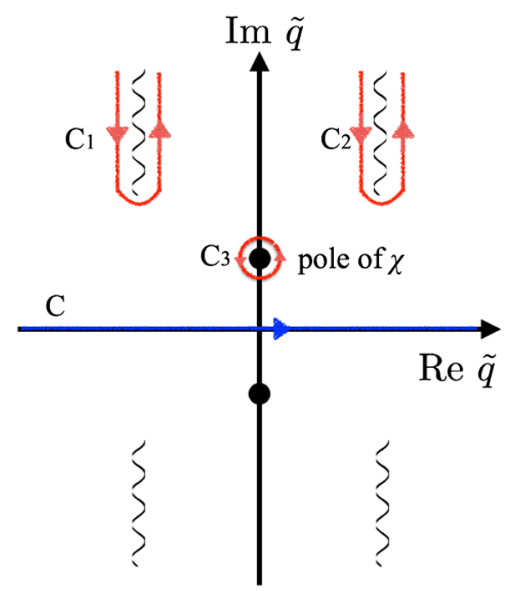

FIG. 1. Singularities of $\chi_{\xi \xi}\left(0, \tilde{q}^{2}\right)$ in the complex- $\tilde{q}$ plane. Cuts are denoted by the wavy lines and poles are denoted by points. The threshold masses correspond to the endpoints of cuts. The original contour $C$ in Eq. (20) is deformed into $C_{1}, C_{2}$ (cut contributions) and $C_{3}$ (pole contribution). For the definition of threshold masses, see Eq. (23).

NJL-type effective models have two problems in the calculation of Eq. (20). The first problem stems from the regularization. The three-dimensional-momentum cutoff regularization commonly used explicitly breaks Lorentz invariance, and induces unphysical oscillations in $\zeta_{\xi \xi}(0, \boldsymbol{x})$ [48]. This problem can be solved by taking the PV regularization [49]. We then use the PV regularization in this paper. The second problem is the fact that direct numerical calculations of $\tilde{q}$ integral is quite difficult because the integrand is highly oscillating at large $r$ where $M_{\xi}^{\text {scr }}$ is defined. In order to overcome this problem, one can rewrite the $\tilde{q}$ integral to the complex $\tilde{q}$ integral by using the Cauchy's integral theorem. However, it is shown in Ref. [48] that the complex function $\chi_{\xi \xi}\left(0, \tilde{q}^{2}\right)$ has logarithmic cuts in the vicinity of the real $\tilde{q}$ axis. The evaluation of the cuts still demands time-consuming numerical calculations. Our previous works $[43,44]$ showed that the emergence of these logarithmic cuts is avoidable by making the $\boldsymbol{p}$ integration analytically before taking the Matsubara (n) summation in Eqs. (12)-(18).

Consequently, we obtain the regularized function $I_{3}^{\text {reg }}$ as an infinite series of analytic functions:

$$
\begin{aligned}
I_{3}^{\mathrm{reg}}\left(0, \tilde{q}^{2}\right)= & i T \sum_{j=1}^{N_{c}} \sum_{n=-\infty}^{\infty} \sum_{\alpha=0}^{2} C_{\alpha} \\
& \times \int \frac{d^{3} \boldsymbol{p}}{(2 \pi)^{3}}\left[\frac{1}{\boldsymbol{p}^{2}+\mathcal{M}^{2}} \frac{1}{(\boldsymbol{p}+\boldsymbol{q})^{2}+\mathcal{M}^{2}}\right] \\
= & \frac{T}{8 \pi \tilde{q}} \sum_{j, n, \alpha} C_{\alpha} \log \left(\frac{2 \mathcal{M}+i \tilde{q}}{2 \mathcal{M}-i \tilde{q}}\right)
\end{aligned}
$$

with a complex valued thermal mass

$$
\mathcal{M}\left(M_{\alpha}, \omega_{n}, A_{4}^{j j}, \mu\right)=\sqrt{M_{\alpha}^{2}+\left(\omega_{n}+A_{4}^{j j}-i \mu\right)^{2}},
$$

where we take the principle value for logarithm in Eq. (21) and the square root in Eq. (22). Each term in the last line of Eq. (21) has four cuts starting at $\tilde{q}=$ $\pm 2 i \mathcal{M}\left(M_{\alpha}, \omega_{n}, A_{4}^{j j}, \mu\right)$ and $\tilde{q}= \pm 2 i \mathcal{M}\left(M_{\alpha}, \omega_{n},-A_{4}^{j j},-\mu\right)$, as shown in Fig. 1. For later convenience, we define the threshold mass $M_{\text {th }}$ and the decay width $\Gamma_{\text {th }}$ by the $\mathcal{M}$ located at the lowest branch point in the upper-half plane: Namely

$$
2 \mathcal{M}_{\text {lowest }} \equiv M_{\text {th }}-i \frac{\Gamma_{\text {th }}}{2},
$$

where $M_{\mathrm{th}}\left(\Gamma_{\mathrm{th}}\right)$ is the real (imaginary) part of $2 \mathcal{M}_{\text {lowest }}$. Meson screening mass $M_{\xi}^{\text {scr }}$ is a pole of $\chi_{\xi \xi}$ and is calculated by

$$
\left.\left[1-2 G_{\mathrm{S}}(\Phi) \Pi_{\xi}\left(0, \tilde{q}^{2}\right)\right]\right|_{\tilde{q}=i M_{\tilde{\xi}}^{\mathrm{scr}}}=0,
$$

when the pole is located below the lowest branch point. This condition leads to [43]

$$
M_{\xi}^{\mathrm{scr}} \leq M_{\mathrm{th}}
$$

\section{NUMERICAL RESULTS}

\section{A. Determination of $T_{0}$}

The EPNJL model has three parameters $T_{0}, \alpha_{1}, \alpha_{2}$. These values are determined so as to reproduce LQCD data [4547] on the chiral and deconfinement transition temperatures $T_{\mathrm{c}}^{\chi, \mathrm{LQCD}} \simeq T_{\mathrm{c}}^{\mathrm{d}, \mathrm{LQCD}}=173 \pm 8 \mathrm{MeV}$. Here the parameters $\alpha_{1}, \alpha_{2}$ are fixed to $\alpha_{1}=\alpha_{2}=0.2$, since the values are necessary to explain the coincidence between the two transitions [39]: namely, $T_{\mathrm{c}}^{\chi, \mathrm{LQCD}} \simeq T_{\mathrm{c}}^{\mathrm{d}, \mathrm{LQCD}}$. The remaining parameter $T_{0}$ is controlled by the absolute values and their errors of $T_{\mathrm{c}}^{\chi, \mathrm{LQCD}}$ and $T_{\mathrm{c}}^{\mathrm{d}, \mathrm{LQCD}}$. The resulting allowed region of $T_{0}$ is constrained into $186 \mathrm{MeV} \leq T_{0} \leq 205 \mathrm{MeV}$. Hereafter we estimate the uncertainty of model prediction by considering the lower and upper limits of $T_{0}$. For later convenience, we also define Set 3 with $T_{0}=200 \mathrm{MeV}$ at which the calculated deconfinement-transition temperature $T_{\mathrm{c}}^{\mathrm{d}, \mathrm{EPNJL}}$ is close to the mean value of $T_{\mathrm{c}}^{\mathrm{d}, \mathrm{LQCD}}$. Eventually, we consider the following three parameter sets;

Set 1: $T_{0}=186 \mathrm{MeV}, \alpha_{1}=\alpha_{2}=0.2$.

Set 2: $T_{0}=205 \mathrm{MeV}, \alpha_{1}=\alpha_{2}=0.2$.

Set 3: $T_{0}=200 \mathrm{MeV}, \alpha_{1}=\alpha_{2}=0.2$.

Figure 2 shows $T$ dependence of $\sigma$ and $|\Phi|$ for the case of $\theta=0$. The model uncertainty is represented by bands for $\sigma$ and $\Phi$. The EPNJL results quantitatively simulate LQCD data on the chiral condensate [45] within the uncertainty of $T_{0}$. As for the Polyakov loop, the EPNJL results agree with LQCD ones [46] in $T \lesssim T_{\mathrm{c}}^{\chi}$, but overestimate them in 


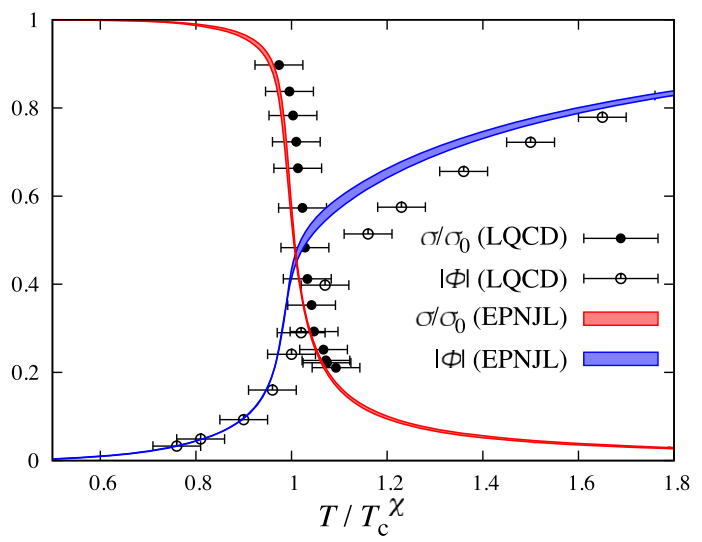

FIG. 2. $T$ dependence of the chiral condensate $\sigma$ and the absolute value $|\Phi|$ of Polyakov loop for $\theta=0$. The horizontal axis is scaled by $T_{\mathrm{c}}^{\chi}$. The $T_{0}$ uncertainty is denoted by bands for $\sigma$ and $\Phi$. The chiral condensate is normalized by its value $\sigma_{0}$ at $T=0$. LQCD data are taken from Refs. $[46,47]$. The $10 \%$ errors come from those of $T_{\mathrm{c}}^{\mathrm{d}, \mathrm{LQCD}}$ and $T_{\mathrm{c}}^{\chi, \mathrm{LQCD}}$.

$1<T / T_{\mathrm{c}}^{\chi}<1.8$. This is the well-known problem in PNJLtype effective models [23,50-52], and it has not been solved yet. However, the discrepancy does not affect the qualitative behavior of $M_{\pi}^{\text {scr }}(\theta)$ and $M_{\sigma}^{\text {scr }}(\theta)$; therefore, we will not discuss the problem in the present work.

\section{B. Deconfinement- and chiral-transition lines in $\theta-T$ plane}

Figure 3 shows the deconfinement- and chiral-transition lines in the imaginary- $\mu$ region. The $T_{0}$ uncertainty mentioned above is represented by the bands. The critical endpoint (CEP) exists on the deconfinement-transition line in both Set 1 and Set 2. For further description, we have varied $T_{0}$ in the allowed region and investigated whether the existence of CEP is robust or not for the $T_{0}$ uncertainty. It is confirmed that the CEP exists for any $T_{0}$ and its location is limited in $0.087 \leq|\theta-\pi / 3| \leq 0.107$, whose range is denoted by two bars in Fig. 3 .

Once one fixes the value of $T_{0}, \theta$ dependence of the transition lines are well fitted in $0 \leq \theta \leq \pi / 3$ by using

$$
\frac{T_{\mathrm{c}}^{\mathrm{X}}(\theta)}{T_{\mathrm{c}}^{\mathrm{X}}(0)}=1+c_{1}^{\mathrm{X}} \theta^{2}+c_{2}^{\mathrm{X}} \theta^{4}
$$

where the superscript " $\mathrm{X}=\mathrm{d}$ " means the deconfinement transition and " $\mathrm{X}=\chi$ " corresponds to the chiral transition. The fitting results for the coefficients and the CEP location $\left(\theta_{\mathrm{CEP}}, T_{\mathrm{CEP}} / T_{\mathrm{c}}^{\chi}(0)\right)$ are summarized in Table II. The fitting results weakly depend on $T_{0}$ and the deviations between Set 1 and Set 2 are less than 10\%.

\section{C. $\theta$ dependence of $\boldsymbol{\pi}$ - and $\boldsymbol{\sigma}$-meson screening masses}

We have confirmed that $\pi$ - and $\sigma$-meson screening masses have the RW periodicity and charge symmetry:

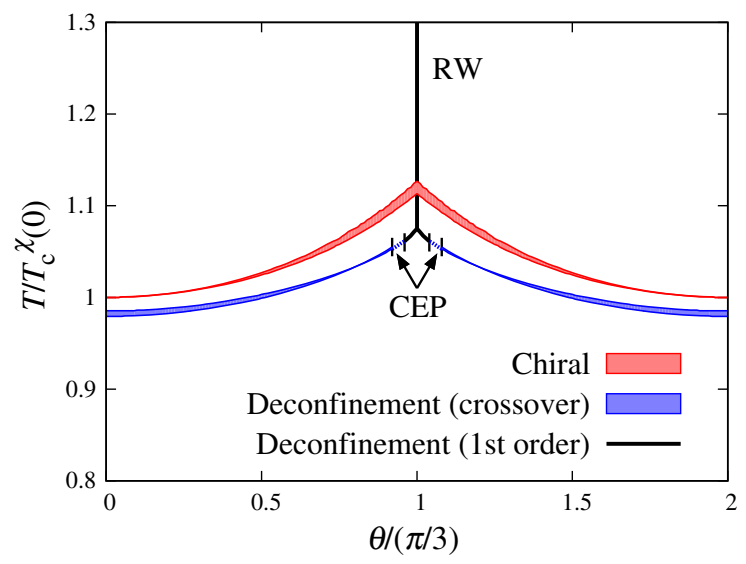

FIG. 3. Deconfinement- and chiral-transition lines in the imaginary $\mu$ region. The thin solid lines with bands stand for the deconfinement- and chiral-transition lines, where the bands are originated in the ambiguity of $T_{0}$. The location of CEP in the deconfinement transition is limited in two bars because of the uncertainty of $T_{0}$. At the CEP, the deconfinement transition is the second order. The first-order RW transition line is denoted by the thick solid line.

$M_{\xi}^{\mathrm{scr}}(\theta)=M_{\xi}^{\mathrm{scr}}\left(\theta+\frac{2 \pi k}{3}\right), \quad M_{\xi}^{\mathrm{scr}}(\theta)=M_{\xi}^{\mathrm{scr}}(-\theta)$

for $\xi=\pi, \sigma$, where $k$ is an arbitrary integer. This result stems from the fact that Eqs. (15)-(18) and the threshold mass $M_{\text {th }}$ are invariant under the extended $Z_{3}$ transformation defined by Eq. (7).

From now on, we consider the following two cases:

(A) $T=1.4 T_{\mathrm{c}}^{\chi}(0)$ in Fig. 3: The system is in both the deconfinement and the chiral-symmetry restored phase for any $\theta$, since $T \geq T_{\mathrm{c}}^{\chi}(\pi / 3)$.

TABLE II. Coefficients $c_{1}^{\mathrm{X}}, c_{2}^{\mathrm{X}}$ and the CEP location for deconfinement- and the chiral-transition lines.

\begin{tabular}{lcc}
\hline \hline & Set $1: \begin{array}{l}T_{0}=186 \mathrm{MeV} \\
\mathrm{X}=\chi\end{array}$ & $\mathrm{X}=\mathrm{d}$ \\
\hline$T_{\mathrm{c}}^{\mathrm{X}}(0)[\mathrm{MeV}]$ & 168 & 165 \\
$c_{1}^{\mathrm{X}}$ & 0.083 & 0.057 \\
$c_{2}^{\mathrm{X}}$ & 0.018 & 0.019 \\
$\left(\theta_{\mathrm{CEP}}, T_{\mathrm{CEP}} / T_{\mathrm{c}}^{\chi}(0)\right)$ & $\cdots$ & $(\pi / 3 \pm 0.042,1.062)$
\end{tabular}

Set 2: $T_{0}=205 \mathrm{MeV}$

$\begin{array}{lcc} & \mathrm{X}=\chi & \mathrm{X}=\mathrm{d} \\ T_{\mathrm{c}}^{\mathrm{X}}(0)[\mathrm{MeV}] & 181 & 177 \\ c_{1}^{\mathrm{X}} & 0.092 & 0.067 \\ c_{2}^{\mathrm{X}} & 0.021 & 0.018 \\ \left(\theta_{\mathrm{CEP}}, T_{\mathrm{CEP}} / T_{\mathrm{c}}^{\chi}(0)\right) & \cdots & (\pi / 3 \pm 0.084,1.055)\end{array}$

Set 3: $T_{0}=200 \mathrm{MeV}$

$\begin{array}{cc}\mathrm{X}=\chi & \mathrm{X}=\mathrm{d} \\ 177 & 174 \\ 0.090 & 0.064 \\ 0.020 & 0.019\end{array}$

\begin{tabular}{lcc}
$T_{\mathrm{c}}^{\mathrm{X}}(0)[\mathrm{MeV}]$ & 177 & 174 \\
$c_{1}^{\mathrm{X}}$ & 0.090 & 0.064 \\
$c_{2}^{\mathrm{X}}$ & 0.020 & 0.019 \\
$\left(\theta_{\mathrm{CEP}}, T_{\mathrm{CEP}} / T_{\mathrm{c}}^{\chi}(0)\right)$ & $\cdots$ & $(\pi / 3 \pm 0.063,1.059)$ \\
\hline \hline
\end{tabular} 


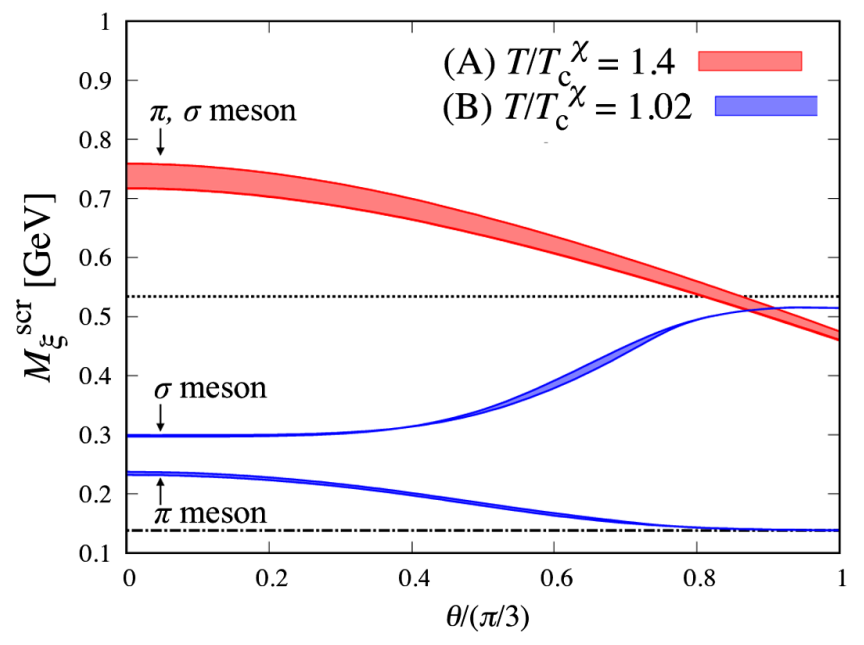

FIG. 4. $\theta$ dependence of $M_{\pi}^{\text {scr }}$ and $M_{\sigma}^{\text {scr }}$ for two cases (A) and (B). The $M_{\pi}^{\text {scr }}$ and $M_{\sigma}^{\text {scr }}$ are shown by the thin solid lines with bands. The dotted line (dot-dash line) denotes $\sigma$-meson ( $\pi$-meson) screening mass at vacuum.

(B) $T=1.02 T_{\mathrm{c}}^{\chi}(0)$ in Fig. 3: This $T$ satisfies $T_{\mathrm{c}}^{\chi}(0) \leq$ $T \leq T_{\mathrm{RW}}$. The system is in the deconfinement phase for $0 \leq \theta \lesssim 0.7$, but in the confinement phase for $0.7 \lesssim \theta \leq \pi / 3$. The system is in the chiral-symmetry restored phase for $0 \leq \theta \lesssim 0.45$ but in the chiralsymmetry broken phase for $0.45 \lesssim \theta \leq \pi / 3$.

Figure 4 explains $\theta$ dependence of $\pi$ - and $\sigma$-meson screening masses for two cases (A) and (B). The $M_{\pi}^{\text {scr }}$ and $M_{\sigma}^{\text {scr }}$ monotonically change as $\theta$ increases for cases (A) and (B). As for case (A), the $\pi$ - and $\sigma$-meson screening masses agree with each other, because of the chiral symmetry restoration. For each of $M_{\pi}^{\text {scr }}$ and $M_{\sigma}^{\text {scr }}$, the deviation between Set 1 and Set 2 is less than $10 \%$ and gets smaller for larger $\theta$.

\section{Fitting of $\pi$ - and $\sigma$-meson screening masses}

In the next subsection, we will extrapolate the meson screening masses from $\mu=i \mu_{\mathrm{I}}$ to $\mu=\mu_{\mathrm{R}}$. For this purpose, we first fit our model results with the polynomial function,

$$
\frac{M_{\xi}^{\mathrm{scr}}\left(T, i \mu_{\mathrm{I}}\right)}{T}=\sum_{n=0}^{n_{\max }} a_{\xi}^{(n)}(T) \theta^{2 n}
$$

in $0 \leq \theta \leq \pi / 3$. We take $n_{\max }=1,2,3,4$ in order to confirm convergence of the expansion. In this procedure, $\theta$ is varied in the range $0 \leq \theta \leq \pi / 3$, although $T$ is fixed. Here the coefficient $a_{\xi}^{(0)}$ is fixed to the $M_{\xi}^{\text {scr }} / T$ at $\theta=0$ and the other coefficients are determined with the least-square method for each $n_{\max }$. From now on, we only consider Set 3 and skip the discussion for the uncertainty of $T_{0}$, since the uncertainty of $T_{0}$ gets smaller for lager $\theta$ and the fixing of $a_{\xi}^{(0)}$ at $\theta=0$ reduces its uncertainty. The resulting $a_{\xi}^{(n)}$ are tabulated in Table III.
TABLE III. Resultant coefficients $a_{\pi}^{(n)}$ and $a_{\sigma}^{(n)}$ for Set 3.

\begin{tabular}{lccccc}
\hline \hline \multicolumn{5}{c}{$T=1.4 T_{\mathrm{c}}^{\chi}(0)$} \\
$n_{\max }$ & $a_{\pi}^{(0)}$ & $a_{\pi}^{(1)}$ & $a_{\pi}^{(2)}$ & $a_{\pi}^{(3)}$ & $a_{\pi}^{(4)}$ \\
\hline 1 & 3.009 & -1.187 & $\ldots$ & $\ldots$ & $\ldots$ \\
2 & 3.009 & -1.453 & 0.343 & $\ldots$ & $\ldots$ \\
3 & 3.009 & -1.542 & 0.638 & -0.214 & $\ldots$ \\
4 & 3.009 & -1.573 & 0.825 & -0.533 & 0.163 \\
& & $T=1.02 T_{\mathrm{c}}^{\chi}(0)$ & & \\
$n_{\max }$ & $a_{\pi}^{(0)}$ & $a_{\pi}^{(1)}$ & $a_{\pi}^{(2)}$ & $a_{\pi}^{(3)}$ & $a_{\pi}^{(4)}$ \\
1 & 1.303 & -0.704 & $\ldots$ & $\ldots$ & $\ldots$ \\
2 & 1.303 & -1.319 & 0.793 & $\ldots$ & $\ldots$ \\
3 & 1.303 & -1.430 & 1.160 & -0.266 & $\ldots$ \\
4 & 1.303 & -1.318 & 0.482 & 0.893 & -0.593 \\
$n_{\max }$ & $a_{\sigma}^{(0)}$ & $a_{\sigma}^{(1)}$ & $a_{\sigma}^{(2)}$ & $a_{\sigma}^{(3)}$ & $a_{\sigma}^{(4)}$ \\
1 & 1.653 & 1.369 & $\ldots$ & $\ldots$ & $\ldots$ \\
2 & 1.653 & 1.520 & -0.196 & $\ldots$ & $\ldots$ \\
3 & 1.653 & -0.0314 & 4.952 & -3.727 & $\ldots$ \\
4 & 1.653 & -0.742 & 9.276 & 11.127 & 3.788 \\
\hline \hline
\end{tabular}

In fact, $\theta$ dependence of $\pi$ - and $\sigma$-meson screening masses is well fitted with the polynomial function (28) with $n_{\max }=4$, as shown in Fig. 5.

\section{E. Extrapolation from $\mu_{\mathrm{I}}$ to $\mu_{\mathrm{R}}$ region}

We compare the extrapolating result with the direct one for finite $\mu_{\mathrm{R}}$ in order to confirm applicability of the analytic continuation. One can easily make the analytic continuation by replacing $\theta$ with $-i \mu_{\mathrm{R}} / T$ :

$$
\frac{M_{\xi}^{\mathrm{scr}}\left(T, \mu_{\mathrm{R}}\right)}{T}=\sum_{n=0}^{n_{\max }}(-1)^{n} a_{\xi}^{(n)}(T)\left(\frac{\mu_{\mathrm{R}}}{T}\right)^{2 n} .
$$

Figure 6 explains $\mu_{\mathrm{R}}$ dependence of $\pi$-meson screening masses for two cases (A) and (B). In $\mu_{\mathrm{R}} / T \lesssim 0.4$, the $M_{\pi}^{\text {scr }}$ converge to the direct results as $n_{\max }$ increases for both the two cases.

Figure 7 shows $\mu_{\mathrm{R}} / T$ dependence of $M_{\sigma}^{\text {scr }}$ for case (B), i.e., $T=1.02 T_{\mathrm{c}}^{\chi}$. We skip case (A) since chiral symmetry is restored in case (A), and $\theta$ dependence of $M_{\sigma}^{\text {scr }}$ is almost

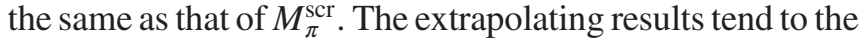
direct ones for $0 \leq \mu_{\mathrm{R}} / T \lesssim 0.4$, and the deviation in $0.4 \leq$ $\mu_{\mathrm{R}} / T$ cannot be improved by taking the higher order terms.

The origin of the deviation can be understood when one considers the relation between $\sigma$-meson screening mass and chiral susceptibility. Equation (19) indicates that the inverse of $M_{\sigma}^{\text {scr }}$ corresponds to the correlation length in the fluctuation of $\langle\bar{\psi}(x) \psi(x)\rangle$; see Ref. [53] for the further explanation, and note that screening mass is referred to be the frequency of "sound mode" there. Hence $M_{\sigma}^{\text {scr }}$ is related to the chiral susceptibility $\chi_{\sigma}$ as 

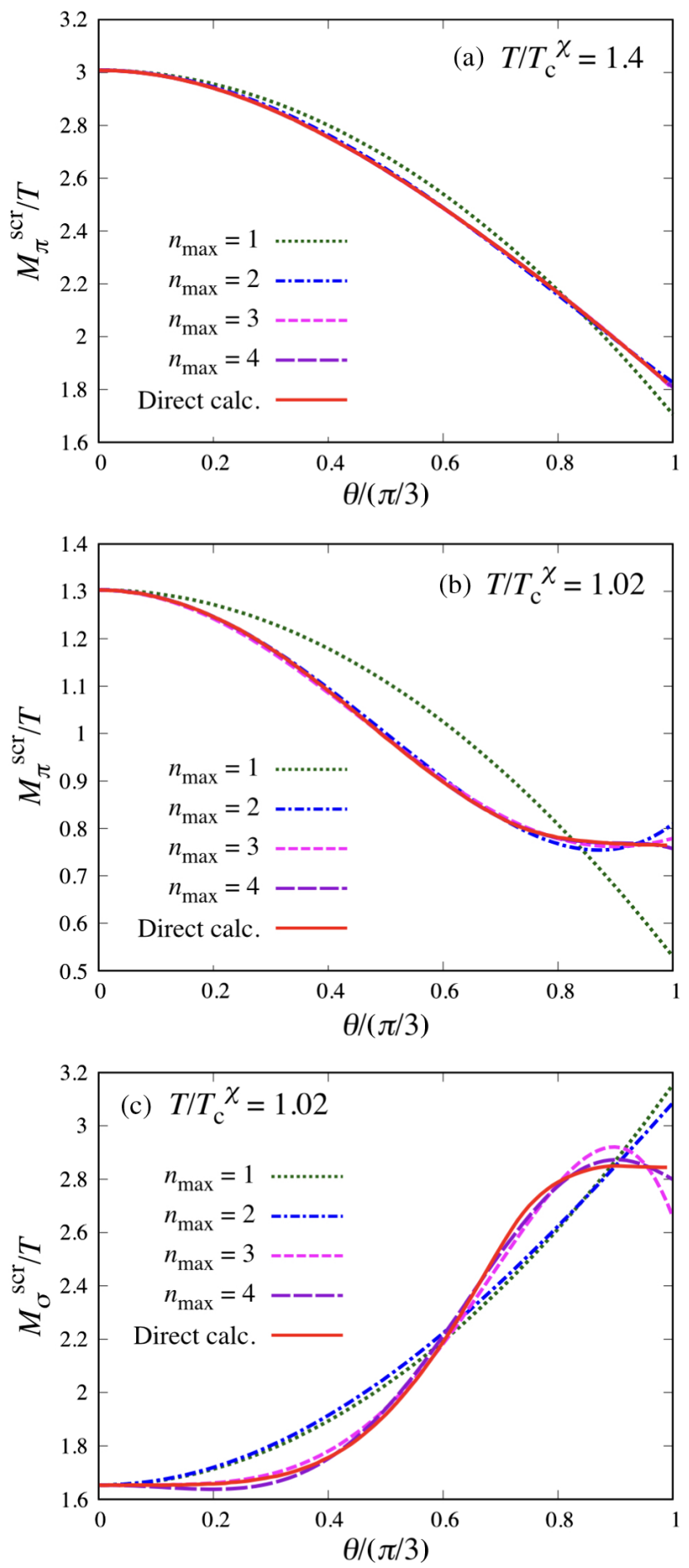

FIG. 5. Polynomial fitting for $\theta$ dependence of $M_{\pi}^{\text {scr }}$ and $M_{\sigma}^{\text {scr }}$ in case (A) and case (B).

$$
M_{\sigma}^{\mathrm{scr}} \propto \chi_{\sigma}^{-1 / 2}
$$

Particularly for the chiral limit, $\mu_{\mathrm{R}}$ and $\mu_{\mathrm{I}}$ dependence of $M_{\sigma}^{\text {scr }}$ is nonanalytic on the chiral-transition line in $\mu_{\mathrm{R}}-T$ and $\mu_{\mathrm{I}}-T$ plane, since $\chi_{\sigma}$ is nonanalytic on the chiral-transition line. As for finite quark mass, a remnant of the nonanalyticity makes the accuracy of the analytic continuation less accurate.
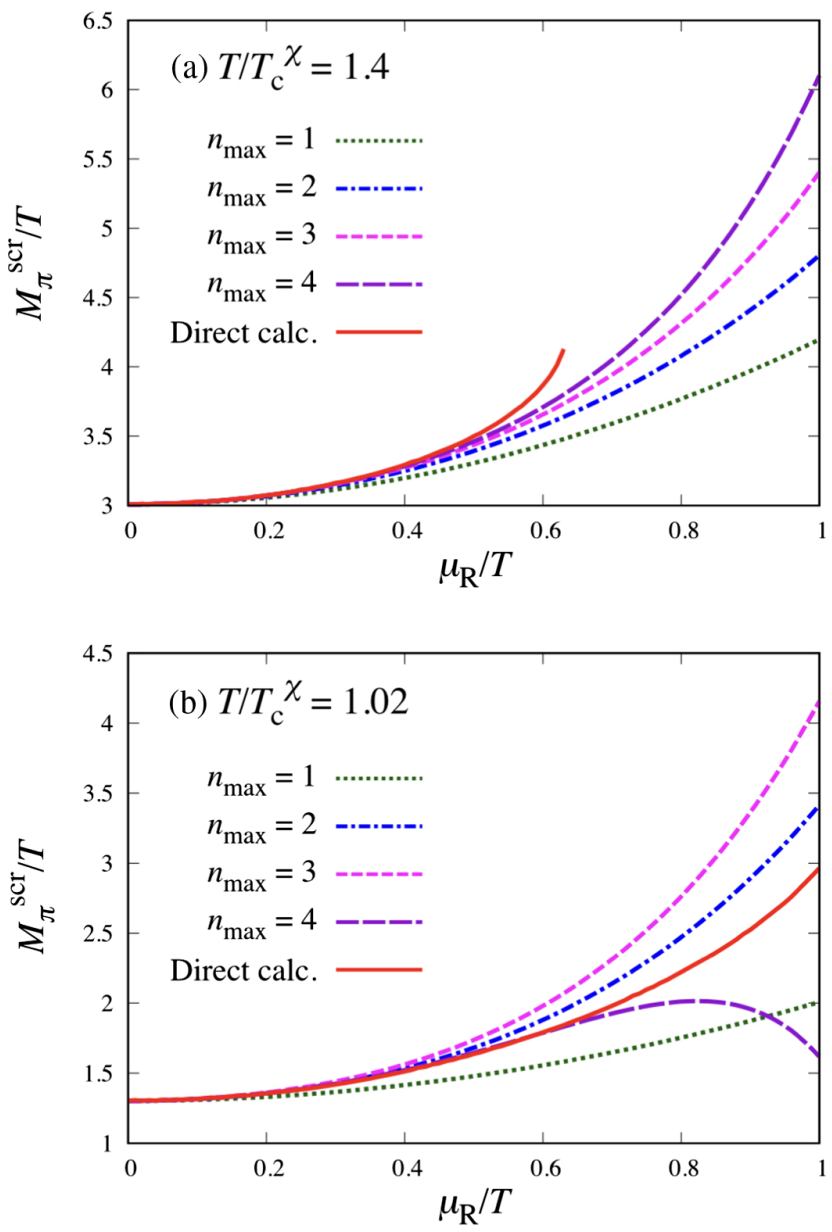

FIG. 6. Comparison between the extrapolating and the direct results on $\mu_{\mathrm{R}} / T$ dependence of $M_{\pi}^{\text {scr }}$. We draw direct-result lines only when the inequality $M_{\pi}^{\text {scr }}<M_{\text {th }}$ in Eq. (25) is satisfied.

\section{F. Phase-transition-line extrapolation}

We propose the new extrapolation method by modifying a trajectory of $(T, \theta)$ in fitting. In standard extrapolation, $\theta$ is varied with fixed $T$. In a new method, we consider to

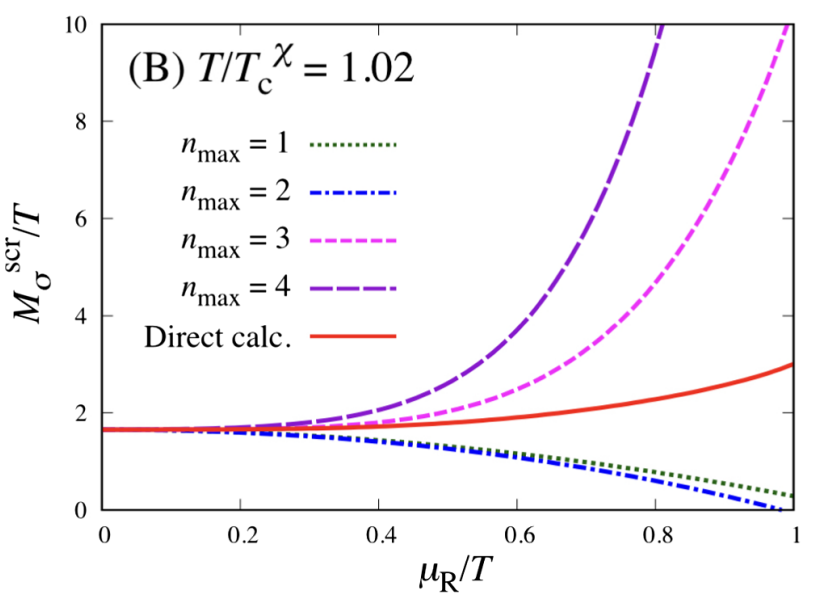

FIG. 7. Comparison between the extrapolating and the direct results on $\mu_{\mathrm{R}} / T$ dependence of $M_{\sigma}^{\mathrm{scr}}$ in case (B), i.e., $T=1.02 T_{\mathrm{c}}^{\chi}$. 


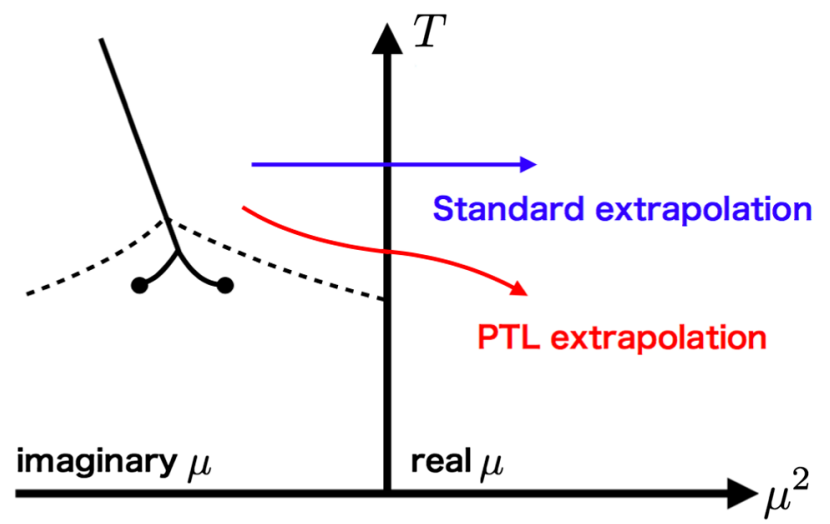

FIG. 8. A schematic figure of PTL extrapolation and standardextrapolation. The arrows stand for the standard extrapolation and the PTL extrapolation. Transition line for chiral symmetry restoration is denoted by the dotted line.

vary $T$ so that the trajectory runs along the chiral-transition line. We then assume $\theta$ dependence of $T$ as

$$
T=T_{\mathrm{PTL}}^{\chi}(\theta)=R \times T_{\mathrm{c}}^{\chi}(\theta)
$$

with any constant $R$ that is introduced to cover the $\theta-T$ plane; see Fig. 8 for the understanding. In this paper, we refer to the modified extrapolation as "phase-transition-line (PTL) extrapolation".

We fit $\theta$ dependence of $\sigma$-meson screening masses with a polynomial series:

$$
\frac{M_{\sigma}^{\mathrm{scr}}(\theta)}{T_{\mathrm{PTL}}^{\chi}(\theta)}=\sum_{n=0}^{n_{\max }} b_{\sigma}^{(n)}(R) \theta^{2 n}
$$

In Eq. (31), the extrapolation line does not pass through the chiral transition line, we can use all range of $\theta$ for fitting, i.e., $0 \leq \theta \leq \pi / 3$.

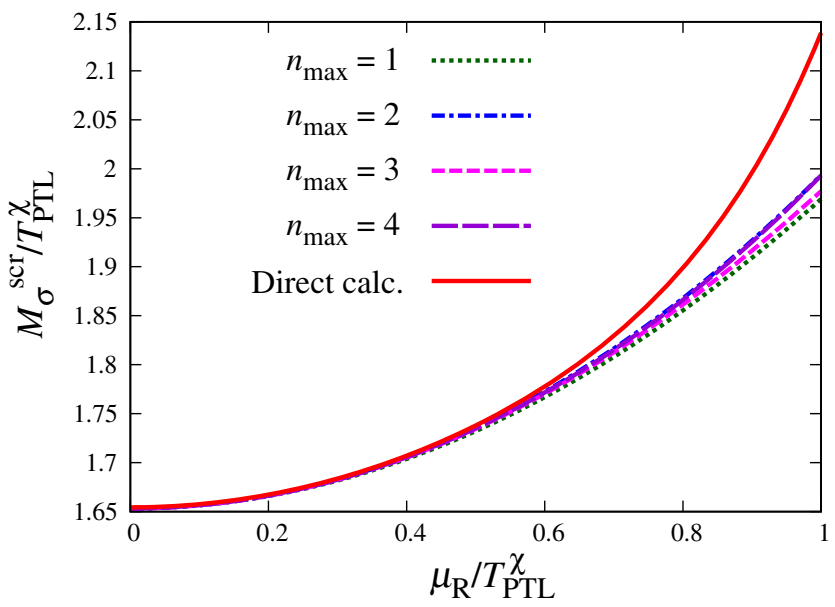

FIG. 9. $\mu_{\mathrm{R}} / T_{\mathrm{PTL}}^{\chi}$ dependence of $\sigma$-meson screening mass for $T_{\text {PTL }}^{\chi}(0)=1.02 T_{\mathrm{c}}^{\chi}(0)$.
TABLE IV. Coefficients $b_{\xi}^{(n)}$ and convergence radii $r_{\xi}^{n_{\max }}$ for $\xi=\pi, \sigma$ with $n_{\max }=1,2,3,4$.

\begin{tabular}{lcccccc}
\hline \hline$n_{\max }$ & $b_{\pi}^{(0)}$ & $b_{\pi}^{(1)}$ & $b_{\pi}^{(2)}$ & $b_{\pi}^{(3)}$ & $b_{\pi}^{(4)}$ & $r_{\pi}^{n_{\max }}$ \\
\hline 1 & 1.303 & -0.270 & $\ldots$ & $\ldots$ & $\ldots$ & 2.196 \\
2 & 1.303 & -0.332 & 0.080 & $\ldots$ & $\ldots$ & 2.042 \\
3 & 1.303 & -0.355 & 0.157 & -0.056 & $\ldots$ & 1.676 \\
4 & 1.303 & -0.360 & 0.187 & -0.107 & 0.026 & 2.018 \\
$n_{\max }$ & $b_{\sigma}^{(0)}$ & $b_{\sigma}^{(1)}$ & $b_{\sigma}^{(2)}$ & $b_{\sigma}^{(3)}$ & $b_{\sigma}^{(4)}$ & $r_{\sigma}^{n_{\max }}$ \\
1 & 1.653 & -0.316 & $\ldots$ & $\ldots$ & $\ldots$ & 2.288 \\
2 & 1.653 & -0.326 & 0.014 & $\ldots$ & $\ldots$ & 4.874 \\
3 & 1.653 & -0.324 & 0.006 & 0.006 & $\ldots$ & 0.986 \\
4 & 1.653 & -0.325 & 0.001 & -0.001 & 0.004 & 0.628 \\
\hline \hline
\end{tabular}

We then extrapolate $M_{\sigma}^{\mathrm{scr}}(\theta)$ and $T_{\mathrm{PTL}}^{\chi}(\theta)$ from finite $\mu_{\mathrm{I}}$ region to $\mu_{\mathrm{R}}$ region. Figure 9 shows the comparison between the direct results and the extrapolating ones for $M_{\sigma}^{\text {scr }}$, where we set $T_{\mathrm{PTL}}^{\chi}(0)=1.02 T_{\mathrm{c}}^{\chi}(0) \simeq 180 \mathrm{MeV}$. The extrapolating results rapidly converge to direct-calculated ones in $\mu_{\mathrm{R}} / T_{\mathrm{PTL}}^{\chi} \lesssim 0.6$. The PTL extrapolation yields better agreement than the standard extrapolation.

We also check the reliability of extrapolation by estimating the radius of convergence in Eq. (32) based on the d'Alembert ratio test. The coefficients $b_{\pi}^{(n)}$ and $b_{\sigma}^{(n)}$ in Eq. (32) are summarized in Table. IV. The radius of convergence $r_{\xi}^{n_{\max }}$ is calculated by $r_{\xi}^{n_{\max }} \equiv$ $\sqrt{b_{\xi}^{\left(n_{\max }-1\right)} / b_{\xi}^{\left(n_{\max }\right)}}$ for $\xi=\pi, \sigma$. For $\xi=\sigma$, the convergence radius gets smaller as $n_{\max }$ increases and the value with $n_{\max }=4$ is estimated as $r_{\sigma}^{n_{\max }}=0.628$, which is consistent with the upper bound of the agreement region $\mu_{\mathrm{R}} / T_{\mathrm{PTL}}^{\chi} \lesssim 0.6$.

Parallel discussion is possible for $M_{\pi}^{\text {scr }}$, as shown in Fig. 10. We can obtain good agreement between direct results and extrapolating ones for $\mu_{\mathrm{R}} / T_{\mathrm{PTL}}^{\chi} \leq \pi / 3$, whose

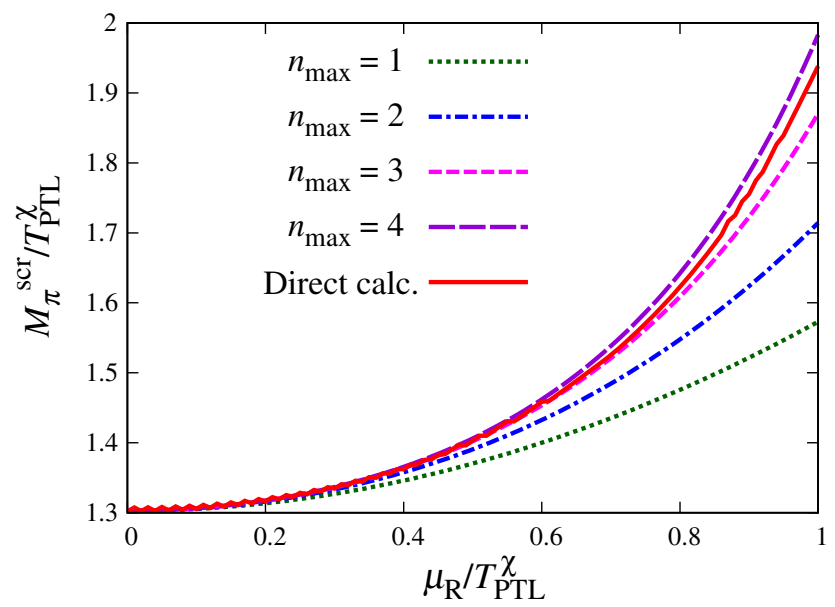

FIG. 10. $\mu_{\mathrm{R}} / T_{\mathrm{PTL}}^{\chi}$ dependence of $\pi$-meson screening mass for $T_{\mathrm{PTL}}^{\chi}(0)=1.02 T_{\mathrm{c}}^{\chi}(0)$. 
region is inside the radius of convergence: $r_{\pi}^{n_{\max }} \sim 2.0$. The value of $r_{\pi}^{n_{\max }}$ is stable as $n_{\max }$ increases.

\section{SUMMARY}

We first showed a method of calculating screening masses for finite $\mu_{\mathrm{R}}$ and $\mu_{\mathrm{I}}$ in the framework of the 2-flavor EPNJL model.

Next, we investigated how reliable the imaginary- $\mu$ approach is for $M_{\pi}^{\text {scr }}$ and $M_{\sigma}^{\text {scr }}$ by comparing "the results extrapolated from imaginary $\mu$ " with "those calculated directly in real $\mu$." In the standard extrapolation, the agreement between the direct and the extrapolating results is seen in $\mu_{\mathrm{R}} / T \lesssim 0.4$ for $M_{\pi}^{\text {scr }}$ and $M_{\sigma}^{\text {scr }}$ for $T=1.02 T_{\mathrm{c}}^{\chi}(0)$ and $T=1.4 T_{\mathrm{c}}^{\chi}(0)$. Especially for $\sigma$ meson, the disagreement in $0.4 \lesssim \mu_{\mathrm{R}} / T$ cannot be improved by taking higher order terms.

We can understand the difficulty of extrapolation when one remembers that $M_{\sigma}^{\text {scr }}$ is nothing but the inverse of correlation length in fluctuation of local chiral condensate. The $M_{\sigma}^{\text {scr }}$ is thus related with the chiral susceptibility $\chi_{\sigma}$ as $M_{\sigma}^{\text {scr }} \propto \chi_{\sigma}^{-1 / 2}$. When one set quark mass to zero, $\chi_{\sigma}$ becomes nonanalytic on the chiral-transition line $T=T_{\mathrm{c}}^{\chi}(\theta)$, and so does $M_{\sigma}^{\text {scr }}$. Even for finite quark mass, a remnant of this nonanalyticity makes the accuracy of extrapolation less accurate, since quark mass is much smaller to temperature and negligible around the chiral phase transition. This indicates that the simple extrapolation is not useful for $M_{\sigma}^{\mathrm{scr}}\left(T, \mu_{\mathrm{R}}\right)$.

In order to circumvent this problem, we propose the PTL extrapolation. In the method, the agreement between the direct and the extrapolating results is seen in $\mu_{\mathrm{R}} / T_{\mathrm{PTL}}^{\chi} \lesssim 0.6$ for $M_{\sigma}^{\text {scr }}$ and in $\mu_{\mathrm{R}} / T_{\mathrm{PTL}}^{\chi} \lesssim \pi / 3$ for $M_{\pi}^{\text {scr }}$ with $T_{\mathrm{PTL}}^{\chi}(0)=1.02 T_{\mathrm{c}}^{\chi}(0)$. The extrapolating results tend to the direct results as higher order terms are taken into account. The PTL extrapolation thus makes better extrapolating results than the standard one.

The difficulty of the simple extrapolation may be in common with other quantities which are sensitive to the chiral transition. The PTL extrapolation is applicable for such quantities except the chiral-transition line itself. This application is quite interesting as a future perspective. For example, $T$ and $\mu$ dependences of $\sigma$ meson screening mass are essential to explore the location of critical endpoint in QCD phase diagram, since $M_{\sigma}^{\mathrm{scr}}(T, \mu)$ goes to zero toward the critical endpoint. Another application is the prediction of meson pole mass from meson screening mass, which is proposed in our previous work [42]. The meson pole mass is one of the experimental observables, and its $T$ and $\mu$ dependences are associated with the ground-state structures of hot QCD matter. In fact, $\eta^{\prime}$ and vector-meson pole masses have been measured in heavy-ion collisions [1,2] in order to investigate the chiral and $U(1)_{\mathrm{A}}$-symmetry restorations. Hence it is important to apply the PTL extrapolation for the determination of $T$ and $\mu$ dependence of light-meson pole masses.

\section{ACKNOWLEDGMENTS}

The authors thank to Kouji Kashiwa and Junpei Sugano for fruitful discussion. M. I., H. K., and M. Y. are supported by Grants-in-Aid for Scientific Research (No. 27-3944, No. 17K05446, and No. 26400278) from the Japan Society for the Promotion of Science (JSPS).

\section{APPENDIX: THE RELATION BETWEEN $A_{4}$ AND $\Phi, \bar{\Phi}$}

The diagonal components $A_{4}^{11}, A_{4}^{22}, A_{4}^{33}$ of the gluon field are related with the Polyakov loop $\Phi$ and its conjugate $\bar{\Phi}$ as

$$
\begin{gathered}
\Phi=\frac{1}{3}\left(\phi_{1}+\phi_{2}+\phi_{3}\right), \\
\bar{\Phi}=\frac{1}{3}\left(\phi_{1}^{*}+\phi_{2}^{*}+\phi_{3}^{*}\right)=\frac{1}{3}\left(\phi_{1} \phi_{2}+\phi_{2} \phi_{3}+\phi_{3} \phi_{1}\right)
\end{gathered}
$$

with $\phi_{j} \equiv \exp \left(i A_{4}^{j j} / T\right) \quad(j=1,2,3)$. Furthermore, the traceless condition for $A_{4}$ leads to

$$
\phi_{1} \phi_{2} \phi_{3}=1 \text {. }
$$

One can confirm that $\phi_{1}, \phi_{1}, \phi_{3}$ are solutions of the following equation:

$$
\phi^{3}-3 \Phi \phi^{2}+3 \bar{\Phi} \phi-1=0
$$

by considering Vieta's formulas. Once we get $\Phi$ and $\bar{\Phi}$, we can obtain $\phi_{1}, \phi_{2}, \phi_{3}$ by solving above equation analytically and get the gluon field as $A_{4}^{j j}=-i T \log \phi_{j}$. The relation between $A_{4}^{j j}$ and $\phi_{j}$ has an ambiguity coming from the replacement $A_{4} \rightarrow A_{4}+2 n \pi T$ for integer $n$, but this ambiguity does not change any physical observables and we simply assume $n=0$. If we take the approximation $\Phi \simeq \bar{\Phi}$, we can simply obtain the gluon fields as

$$
A_{4}^{11}=-A_{4}^{22}=T \cos ^{-1}\left(\frac{3 \Phi-1}{2}\right), \quad A_{4}^{33}=0 .
$$


[1] L. Adamczyk et al. (STAR Collaboration), Phys. Rev. Lett. 113, 022301 (2014).

[2] T. Csörgő, R. Vértesi, and J. Sziklai, Phys. Rev. Lett. 105, 182301 (2010).

[3] C. R. Allton, S. Ejiri, S. J. Hands, O. Kaczmarek, F. Karsch, E. Laermann, Ch. Schmidt, and L. Scorzato, Phys. Rev. D 66, 074507 (2002).

[4] S. Ejiri, Y. Maezawa, N. Ukita, S. Aoki, T. Hatsuda, N. Ishii, K. Kanaya, and T. Umeda (WHOT-QCD Collaboration), Phys. Rev. D 82, 014508 (2010).

[5] Z. Fodor and S. D. Katz, Phys. Lett. B 534, 87 (2002).

[6] P. de Forcrand and O. Philipsen, Nucl. Phys. B642, 290 (2002); B673, 170 (2003);

[7] M. D'Elia and M. P. Lombardo, Phys. Rev. D 67, 014505 (2003); 70, 074509 (2004).

[8] K. Nagata and A. Nakamura, Phys. Rev. D 83, 114507 (2011).

[9] J. Sugano, J. Takahashi, H. Kouno, and M. Yahiro, arXiv:1709.02198.

[10] A. Nakamura, S. Oka, and Y. Taniguchi, J. High Energy Phys. 02 (2016) 054.

[11] G. Aarts, Phys. Rev. Lett. 102, 131601 (2009).

[12] G. Aarts, L. Bongiovanni, E. Seiler, D. Sexty, and I.-O. Stamatescu, Eur. Phys. J. A 49, 89 (2013).

[13] D. Sexty, Phys. Lett. B 729, 108 (2014).

[14] G. Aarts, E. Seiler, D. Sexty, and I.-O. Stamatescu, Phys. Rev. D 90, 114505 (2014).

[15] M. Cristoforetti, F. Di Renzo, and L. Scorzato (AuroraScience Collaboration), Phys. Rev. D 86, 074506 (2012).

[16] H. Fujii, D. Honda, M. Kato, Y. Kikukawa, S. Komatsu, and T. Sano, J. High Energy Phys. 10 (2013) 147.

[17] A. Roberge and N. Weiss, Nucl. Phys. B275, 734 (1986).

[18] P. N. Meisinger and M. C. Ogilvie, Phys. Lett. B 379, 163 (1996).

[19] A. Dumitru and R. D. Pisarski, Phys. Rev. D 66, 096003 (2002).

[20] K. Fukushima, Phys. Lett. B 591, 277 (2004); Phys. Rev. D 77, 114028 (2008); 78, 114019 (2008).

[21] P. Costa, M. C. Ruivo, C. A. de Sousa, and Y. L. Kalinovsky, Phys. Rev. D 71, 116002 (2005).

[22] S. K. Ghosh, T. K. Mukherjee, M. G. Mustafa, and R. Ray, Phys. Rev. D 73, 114007 (2006).

[23] E. Megías, E. R. Arriola, and L. L. Salcedo, Phys. Rev. D 74, 065005 (2006).

[24] C. Ratti, M. A. Thaler, and W. Weise, Phys. Rev. D 73, 014019 (2006).

[25] C. Ratti, S. Rößner, M. A. Thaler, and W. Weise, Eur. Phys. J. C 49, 213 (2007).

[26] S. Rößner, C. Ratti, and W. Weise, Phys. Rev. D 75, 034007 (2007).
[27] H. Hansen, W. M. Alberico, A. Beraudo, A. Molinari, M. Nardi, and C. Ratti, Phys. Rev. D 75, 065004 (2007).

[28] C. Sasaki, B. Friman, and K. Redlich, Phys. Rev. D 75, 074013 (2007).

[29] B.-J. Schaefer, J. M. Pawlowski, and J. Wambach, Phys. Rev. D 76, 074023 (2007).

[30] M. Ciminale, R. Gatto, G. Nardulli, and M. Ruggieri, Phys. Lett. B 657, 64 (2007); M. Ciminale, R. Gatto, N. D. Ippolito, G. Nardulli, and M. Ruggieri, Phys. Rev. D 77, 054023 (2008).

[31] K. Kashiwa, H. Kouno, M. Matsuzaki, and M. Yahiro, Phys. Lett. B 662, 26 (2008).

[32] Y. Sakai, K. Kashiwa, H. Kouno, and M. Yahiro, Phys. Rev. D 77, 051901(R) (2008); 78, 036001 (2008).

[33] Y. Sakai, K. Kashiwa, H. Kouno, M. Matsuzaki, and M. Yahiro, Phys. Rev. D 78, 076007 (2008).

[34] Y. Sakai, K. Kashiwa, H. Kouno, M. Matsuzaki, and M. Yahiro, Phys. Rev. D 79, 096001 (2009).

[35] H. Kouno, Y. Sakai, K. Kashiwa, and M. Yahiro, J. Phys. G 36, 115010 (2009).

[36] P. Costa, M. C. Ruivo, C. A. de Sousa, H. Hansen, and W. M. Alberico, Phys. Rev. D 79, 116003 (2009).

[37] Y. Sakai, T. Sasaki, H. Kouno, and M. Yahiro, J. Phys. G 37, 105007 (2010).

[38] Y. Sakai, T. Sasaki, H. Kouno, and M. Yahiro, Phys. Rev. D 82, 096007 (2010).

[39] Y. Sakai, T. Sasaki, H. Kouno, and M. Yahiro, Phys. Rev. D 82, 076003 (2010).

[40] Y. Sakai, T. Sasaki, H. Kouno, and M. Yahiro, J. Phys. G 39, 035004 (2012).

[41] M. C. Ruivo, M. Santos, P. Costa, and C. A. de Sousa, Phys. Rev. D 85, 036001 (2012).

[42] M. Ishii, H. Kouno, and M. Yahiro, Phys. Rev. D 95, 114022 (2017).

[43] M. Ishii, T. Sasaki, K. Kashiwa, H. Kouno, and M. Yahiro, Phys. Rev. D 89, 071901(R) (2014).

[44] M. Ishii, K. Yonemura, J. Takahashi, H. Kouno, and M. Yahiro, Phys. Rev. D 93, 016002 (2016).

[45] F. Karsch, Lect. Notes Phys. 583, 209 (2002).

[46] O. Kaczmarek and F. Zantow, Phys. Rev. D 71, 114510 (2005).

[47] F. Karsch, E. Laermann, and A. Peikert, Nucl. Phys. B605, 579 (2001).

[48] W. Florkowski, Acta Phys. Pol. B 28, 2079 (1997).

[49] W. Pauli and F. Villars, Rev. Mod. Phys. 21, 434 (1949).

[50] J. Braun, H. Gies, and J. M. Pawlowski, Phys. Lett. B 684, 262 (2010).

[51] F. Marhauser and J. M. Pawlowski, arXiv:0812.1144.

[52] E. R. Arriola, L. L. Salcedo, and E. Megías, Acta Phys. Pol. B 45, 2407 (2014).

[53] H. Fujii, Phys. Rev. D 67, 094018 (2003). 